\title{
Realizing the Unexploited Potential of Games on Serious Challenges
}

\author{
C. E. PALAZZI \\ University of Padua, Italy \\ AND \\ M. ROCCETTI, G. MARFIA \\ University of Bologna, Italy
}

Games are very popular for their entertainment purpose that attracts people of every age and gender. They also represent a very successful industry; leveraging on the wide popularity of games among all the segments of the consumer market, game companies have been able to generate higher revenues than the movie industry.

However, considering games just as a matter of fun and money is definitely wrong. Games also represent a challenging topic for researchers all over the world. One of the reasons is the correlation between problems that emerge in developing innovative game experiences and those typical of other conventional research fields in computer science. We can hence state that games are factually contributing to knowledge advancement.

Moreover, let us consider one of the well known ways through which games contribute to our society. Serious games are applications that couple the pure entertainment with a serious purpose [Michael and Chen 2005]. This feature is generally exploited to educate, train, heal, advertise, and inform. For instance, DroidGlove is a smart phone based game for wrist rehabilitation that provides the user with the task of performing a series of hand movements and verifies the result through the accelerometer sensor [Deponti et al. 2009]. Another interesting example is September 12th that is intended to make people aware about the counter-effects in using violence to resolve the problem of terrorism [Frasca 2010]. We can cite also the very famous Microsoft Flight Simulator, which is used as a first step even to train real pilots.

In essence, the usefulness of serious games is proven by a huge amount of available titles, not just very famous ones, but even very small productions that are created, for instance, to teach kids on proper waste recycle. The magic trick of the serious game approach lies in the fact that a positive aim can be reached through attracting people in an entertaining task. Yet, we argue that the positive development process on our society generated by the serious game paradigm is still very slow, with limited outcomes, not considering automatically generated data, and definitely not exploiting all of its potential.

The real impact of serious games on our society will happen when they will be utilized to gather the contribution of thousands, maybe millions, of player all around the world to address crucial and unsolved problems. In essence, we are waiting for serious games to be combined with crowd-sourcing and mobile users with sensor-equipped smart phones; this will create a major force able to tackle serious challenges that can be considered too complex for single users and even for computers.

The seed of this revolution has been already planted. Think to Google Image Labeler, which exploits game dynamics between two users to overcome the limitation of computers in tagging images [GOOGLE 2007]. Google Image Labeler is a web application that leaves this task to users who have to compete in suggesting tags, until the same tag is proposed by both players; then, that tag is associated to the image. Beside users' fun, the purpose of this game is to create a database of images with appropriate 
tags, overcoming the inability of computers in tagging/recognizing images. Indeed, once integrated with any simple keywords-based search engine, this database allows to simply using textual search keys to retrieve appropriate images. This example shows how serious games can exploit crowd-sourcing to tackle complex issues. Yet, this is not enough.

In fact, even this case is based on the classic game model with static users connected through their PCs; this neglects the enormous potential offered today by the more and more popular smart phones and their sensors (i.e., accelerometer, GPS, microphone, camera).

The horizons of this context can be pushed further by enabling new interconnecting technologies and creating serious games characterized as pervasive. In the era of Web 2.0, with the approaching $\mathrm{Web}^{2}$ advent [O'Reilly and Battelle 2009], more can be done that can be beneficial for the society as a whole. The players' teamwork could produce results that are more valuable than the sum of those achievable by individuals [Ferretti et al. 2010]. Even better, information automatically generated by sensors available on smart phones may integrate the data generated by the community of players, generating new intelligence [Palazzi et al. 2010].

As a first example, we have imagined how a serious game could help those users that are disadvantaged in our society: people with illnesses or disabilities, or elders. Revisiting the aforementioned example, we could extend the idea of Google Image Labeler in a pervasive context where, through their smart phones, users label the surrounding environment (e.g., crossroads, architectural barriers, parks, stores), rather than simple images, creating a participative augmented reality environment. A visually impaired person could then consume this pervasive information through her/his mobile device that is able to retrieve it and to transform its format (text, image, video, etc.) in audio, thus improving her/his autonomy.

If you are not yet convinced, then consider Google Maps. This very useful service can provide path information between any two locations for cars and pedestrians. Unfortunately, it does not provide any information about the accessibility of suggested roads. This is due to the fact that there are no digital data referring to this aspect, thus impeding to algorithms to consider accessibility for people with impairments when generating a path. A classic approach to address this problem would be that of paying somebody to create and continuously update this database. Clearly, on a worldwide scale, this represents a huge cost.

Instead, a much smarter and less expensive idea is to exploit a pervasive serious game where users are requested to take note of architectural impediments and accessibility on the street. By playing a treasure hunt type game, players could be asked to document through pictures a certain number of non-accessible curbs for wheelchairs, or they could be asked to record blind audible traffic signals of as many intersections as possible exploiting their phone's microphone. Then, these data will be integrated with the GPS coordinates of the smart phones and sent to a remote server. Thereby, the reward for players may be game points, free ring tones, or awards from the municipality; but the benefits for the whole society would be to overcome, at almost no cost, the tough challenge of having a continuously updated database with street accessibility information.

These few examples demonstrate how pervasive serious games could be employed to solve very complex issues and improve our society. Yet, as the process of inventing serious games is fun per se, we conclude with a final serious game for you. We challenge your mind to invent as many serious games as possible; then send us your more innovative and beneficial ideas and the best will be rewarded. 


\section{REFERENCES}

MICHAEL, D.R, CHEN, S.L., 2005. Serious games: games that educate, train and inform. Muska and Lipman/Premier-Trade Publisher, 2005.

DEPONTI, D., MAGGIORINI, D., PALAZZI, C. E., 2009. DroidGlove: An Android-Based Application for Wrist Rehabilitation. In Proceedings of IEEE International Conference on Ultramodern Telecommunications (ICUMT 2009), St. Petersburg, Russia, October 2009.

FRASCA G., 2010. NEWSGAMING - SEPTEMBER 12. Available online http://www.newsgaming.com/games/index12.htm

GOOGLE IMAGE LABELER. 2007. Available online http://images.google.com/imagelabeler/

FERRETTI, S., FURINI, M., PALAZZI, C.E., ROCCETTI, M., SALOMONI, P., 2010. WWW recycling for a better world. Communications of the ACM. 53(4), 139-143.

PALAZZI, C.E., TEODORI, L., ROCCETTI, M., 2010. PATH 2.0: a participatory system for the generation of accessible routes. In Proceedings of 2010 IEEE International Conference on Multimedia and Expo, Singapore, July 2010.

O'REILLY, T., BATTELLE, J., 2009. Web Squared: Web Squared: Web 2.0 Five Years On. Available online http://www.web2summit.com/web2009/public/schedule/detail/10194 